\title{
Cosmology with the Planck Satellite: from quantum foam to the cosmic web
}

\author{
François R. Bouchet* ${ }^{*}$ \\ Institut d'Astrophysique de Paris \\ CNRS $\mathcal{E}$ Sorbonne Université-UPMC, \\ 98 bis Boulevard Arago, F-75014, Paris, France \\ E-mail: bouchet@iap.fr
}

\begin{abstract}
Sketched out in 1992, selected by ESA in 1996, and launched in 2009, the Planck satellite was shut off in 2013, after a measuring mission that exceeded all expectations. The Planck collaboration delivered a first set of cosmological data and results in March 21 ${ }^{\text {st }}$ 2013, and the full set in February 2015. Part of the data delivery is a "definitive" map of the anisotropies of the Cosmic Microwave Background (CMB), its angular power spectrum together with their full statistical characterisation. The 2015 delivery also includes pioneering polarisation data. I describe results we obtained so far from temperature and polarisation data, both in terms of content of the universe and of characteristics of the primordial fluctuations. This overview is entirely based on the published or submitted works of the Planck collaboration.
\end{abstract}

The 11th International Workshop Dark Side of the Universe 2015

14-18 December 2015

Kyoto, Japan

\footnotetext{
* Speaker.

${ }^{\dagger}$ Planck results given on behalf of the Planck collaboration.
} 


\section{The Planck mission}

The European Space Agency's Planck satellite ${ }^{1}[43 ; 61]$ is dedicated to studying the early Universe and its subsequent evolution. The satellite was launched on May $14^{\text {th }} 2009$ and scanned the microwave and sub millimetre sky continuously between 12 August 2009 and 23 October 2013. Planck's scientific payload contained an array of 74 detectors in nine frequency bands sensitive to frequencies between 25 and $1000 \mathrm{GHz}$, which scanned the sky with angular resolution between $33^{\prime}$ and $5^{\prime}$. The detectors of the Low Frequency Instrument [LFI; 4; 36] were pseudo-correlation radiometers, covering bands centred at 30,44 , and $70 \mathrm{GHz}$. The detectors of the High Frequency Instrument [HFI; 34; 42] were bolometers, cooled ${ }^{2}$ at $0.1 \pm 0.001 \mathrm{~K}$ and covering bands centred at $100,143,217,353,545$, and $857 \mathrm{GHz}$. Planck imaged the whole sky twice per year, with a combination of sensitivity, angular resolution, and frequency coverage never before achieved. Planck, its payload, and its performance as predicted at the time of launch are described in 13 papers included in a special issue of Astronomy \& Astrophysics (Volume 520). All results obtained so far are discussed in detail in a suite of more than 150 papers by the Planck collaboration. A summary of the characteristics of the LFI and HFI maps based on the data of the full mission is provided in Tables $1 \& 2$. Note in particular the excellent sensitivity achieved by HFI in the core CMB channels, on which most cosmology results rely, of $1.29,0.55,0.78 \mu \mathrm{K}_{\mathrm{CMB}}$ deg at (respectively) 100,143 , and $217 \mathrm{GHz}$. These numbers indicate the rms of the fluctuations contributed by detector noise in pixels of 1 degree on a side (leading to $7.5,4.3,8.7 \mu \mathrm{K}_{\mathrm{CMB}}$ per (Gaussian) beam solid angle of FWHM equal to 9.66, 7.22, 4.90 arcmin (the rms of the CMB anisotropy is about $100 \mu \mathrm{K}_{\mathrm{CMB}}$ ). For white noise (a reasonable approximation at scales below a degree), the detector noise scales inversely proportional to the pixel linear size.

\section{CMB maps from Planck}

The nine all-sky high-sensitivity high angular resolution Planck maps in intensity and their associated polarisation maps at seven frequencies are a treasure trove for astrophysics, which have already allowed many progresses in the understanding of the various astrophysical sources of emission in the millimetre and sub-millimetre range, e.g., on the diffuse Galactic emission (in particular synchrotron, free-free, $\mathrm{CO}$, spinning and thermal dust), as well as compact sources (radio-sources, Infra-red galaxies, Sunyaev-Zeldovich clusters) and the unresolved Cosmic Infra-red background (which is the integrated light from all infrared sources along the line of sight.

\subsection{CMB map cleaning}

In order to clean the background CMB map from foreground emissions, we have used four different approaches (Commander, NILC, SEVEM, SMICA) which combine differently the various

\footnotetext{
${ }^{1}$ Planck (http://www.esa.int/Planck) is a project of the European Space Agency (ESA) with instruments provided by two scientific consortia funded by ESA member states and led by Principal Investigators from France and Italy, telescope reflectors provided through a collaboration between ESA and a scientific consortium led and funded by Denmark, and additional contributions from NASA (USA).

${ }^{2}$ Since the HFI operational temperature of $0.1 \mathrm{~K}$ was achieved thanks to an open loop dilution fridge, the HFI took survey data till the exhaust of the cryogens, i.e. for about 30 months, while the LFI kept taking data for the full duration of about 4 years.
} 
Table 1: Main characteristics of LFI full mission maps.

\begin{tabular}{|c|c|c|c|}
\hline \multirow[b]{2}{*}{ Characteristic } & \multicolumn{3}{|c|}{ Frequency band } \\
\hline & $30 \mathrm{GHz}$ & $44 \mathrm{GHz}$ & $70 \mathrm{GHz}$ \\
\hline Centre frequency $[\mathrm{GHz}] \ldots \ldots \ldots \ldots \ldots \ldots$ & 28.4 & 44.1 & 70.4 \\
\hline Effective beam FWHM ${ }^{\mathrm{a}}$ [arcmin] .. & 32.29 & 27.00 & 13.21 \\
\hline Effective beam ellipticity ${ }^{\mathrm{a}} \ldots \ldots$ & 1.32 & 1.04 & 1.22 \\
\hline Temperature noise $\left(1^{\circ}\right)^{\mathrm{b}}\left[\mu \mathrm{K}_{\mathrm{CMB}}\right] \ldots \ldots \ldots \ldots \ldots$ & 2.5 & 2.7 & 3.5 \\
\hline Polarization noise $\left(1^{\circ}\right)^{\mathrm{b}}\left[\mu \mathrm{K}_{\mathrm{CMB}}\right] \ldots \ldots \ldots \ldots$ & 3.5 & 4.0 & 5.0 \\
\hline Overall calibration uncertainty ${ }^{\mathrm{c}}[\%]$. & 0.35 & 0.26 & 0.20 \\
\hline Systematic effects uncertainty in Stokes $I^{\mathrm{d}}\left[\mu \mathrm{K}_{\mathrm{CMB}}\right] \ldots$ & 0.19 & 0.39 & 0.40 \\
\hline Systematic effects uncertainty in Stokes $Q^{\mathrm{d}}\left[\mu \mathrm{K}_{\mathrm{CMB}}\right] \ldots$ & 0.20 & 0.23 & 0.45 \\
\hline Systematic effects uncertainty in Stokes $U^{\mathrm{d}}\left[\mu \mathrm{K}_{\mathrm{CMB}}\right] \ldots$ & 0.40 & 0.45 & 0.44 \\
\hline
\end{tabular}

${ }^{a}$ Calculated from the main beam solid angle of the effective beam, $\Omega_{\mathrm{eff}}=$ mean $(\Omega)$. These values are used in the source extraction pipeline [54].

${ }^{b}$ Noise rms computed after smoothing to $1^{\circ}$.

${ }^{c}$ Sum of the error determined from the absolute and relative calibration, see [45].

${ }^{d}$ Estimated rms values over the full sky and after full mission integration. Not included here are gain reconstruction uncertainties, estimated to be of order $0.1 \%$.

Table 2: Main characteristics of HFI full mission maps.

\begin{tabular}{|c|c|c|c|c|c|c|c|}
\hline \multirow[b]{2}{*}{ Characteristic } & \multicolumn{6}{|c|}{ Reference frequency $v[\mathrm{GHz}]$} & \multirow[b]{2}{*}{ Notes } \\
\hline & 100 & 143 & 217 & 353 & 545 & 857 & \\
\hline Number of bolometers & 8 & 11 & 12 & 12 & 3 & 4 & a1 \\
\hline Effective beam FWHM ${ }_{1}[\operatorname{arcmin}] \ldots$ & 9.68 & 7.30 & 5.02 & 4.94 & 4.83 & 4.64 & b1 \\
\hline Effective beam $\mathrm{FWHM}_{2}[\operatorname{arcmin}] \ldots$ & 9.66 & 7.22 & 4.90 & 4.92 & 4.67 & 4.22 & b2 \\
\hline Effective beam ellipticity $\epsilon \ldots \ldots$. . & 1.186 & 1.040 & 1.169 & 1.166 & 1.137 & 1.336 & b3 \\
\hline Noise per beam solid angle $\left[\mu \mathrm{K}_{\mathrm{CMB}}\right]$. & 7.5 & 4.3 & 8.7 & 29.7 & & & $\mathrm{c} 1$ \\
\hline$\left[\mathrm{kJy} \mathrm{sr}^{-1}\right]$ & $\ldots$ & $\ldots$ & $\ldots$ & $\ldots$ & 9.1 & 8.8 & $\mathrm{c} 1$ \\
\hline Temperature noise $\left[\mu \mathrm{K}_{\mathrm{CMB}} \operatorname{deg}\right] \ldots$ & 1.29 & 0.55 & 0.78 & 2.56 & $\ldots$ & $\ldots$ & c2 \\
\hline$\left[\mathrm{kJy} \mathrm{sr}^{-1} \mathrm{deg}\right] \ldots$ & $\ldots$ & $\ldots$ & $\ldots$ & $\ldots$ & 0.78 & 0.72 & c2 \\
\hline Polarization noise $\left[\mu \mathrm{K}_{\mathrm{CMB}} \mathrm{deg}\right] \ldots \ldots$ & 1.96 & 1.17 & 1.75 & 7.31 & $\ldots$ & $\ldots$ & c3 \\
\hline Calibration accuracy $[\%]$. & 0.09 & 0.07 & 0.16 & 0.78 & $1.1(+5)$ & $1.4(+5)$ & $\mathrm{d}$ \\
\hline CIB monopole prediction [ $\left.\mathrm{MJy} \mathrm{sr}^{-1}\right]$. & 0.0030 & 0.0079 & 0.033 & 0.13 & 0.35 & 0.64 & $\mathrm{e}$ \\
\hline
\end{tabular}

${ }^{\text {a1 }}$ Number of bolometers whose data were used in producing the channel map.

${ }^{b 1}$ FWHM of the Gaussian whose solid angle is equivalent to that of the effective beams.

b2 FWHM of the elliptical Gaussian fit.

${ }^{\text {b3 }}$ Ratio of the major to minor axis of the best-fit Gaussian averaged over the full sky.

${ }^{\mathrm{c} 1}$ Estimate of the noise per beam solid angle, as given in $b 1$.

${ }^{c 2}$ Estimate of the noise in intensity scaled to $1^{\circ}$ assuming that the noise is white.

${ }^{\mathrm{c} 3}$ Estimate of the noise in polarization scaled to $1^{\circ}$ assuming that the noise is white.

d Calibration accuracy (at 545 and $857 \mathrm{GHz}$, the $5 \%$ accounts for the model uncertainty).

e According to the [5] model, whose uncertainty is estimated to be at the $20 \%$ level (also for constant $v I_{v}$ ). 

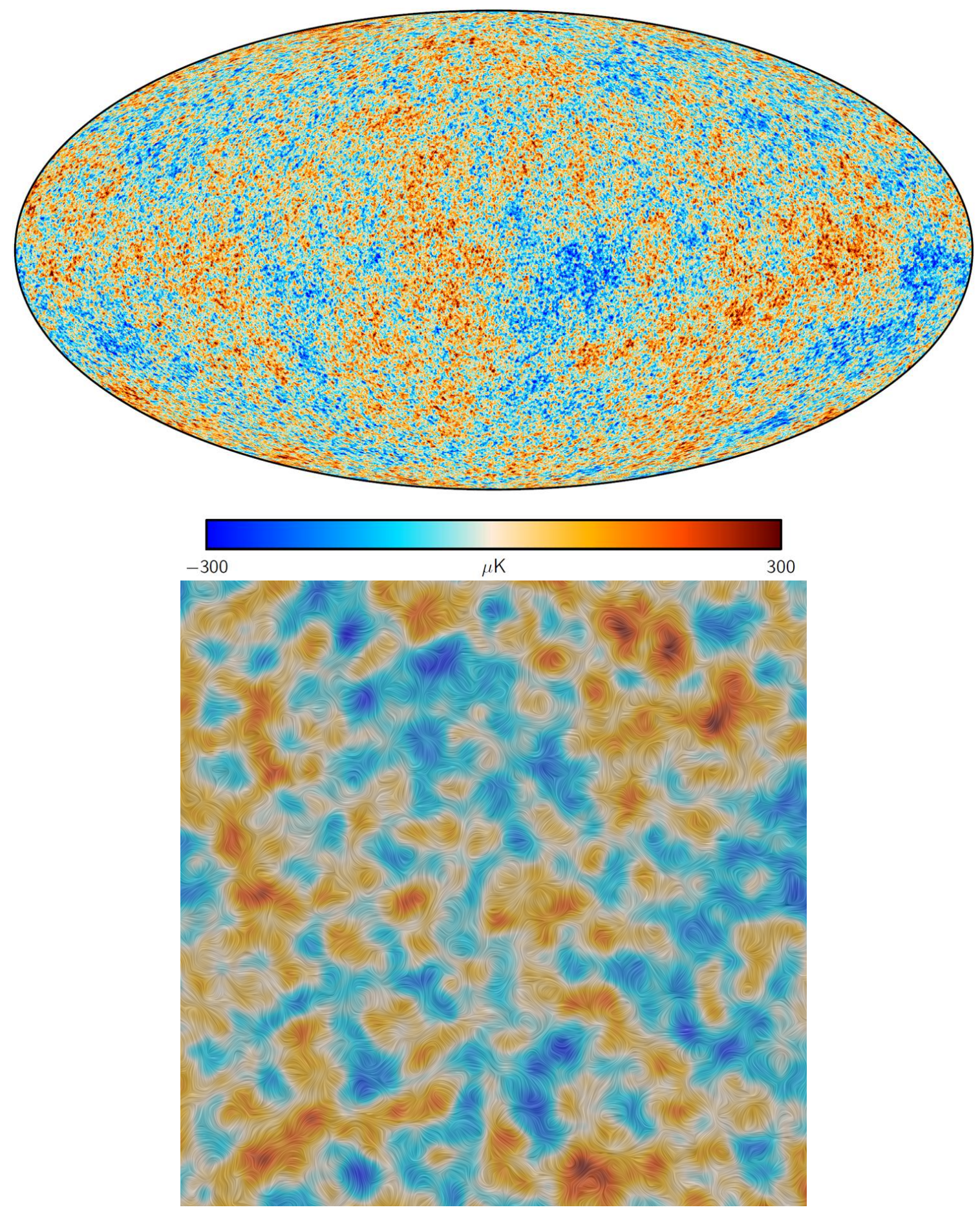

Figure 1: Top: Planck 2015 temperature anisotropies map. A small strip in the direction of the Galactic plane is filled in by a constrained realization that has the same statistical properties as the rest of the sky (this area of the sky is obviously not use for any CMB science analysis). Bottom: Planck 2015 CMB polarisation map zoomed, with 20 arc minute smoothing, revealing smaller scales details (the data is natively at 5 minutes of arc resolution).The coloured background shows the temperature anisotropies, enabling to visually perceive the correlation between the temperature and polarisation fields. 
frequency maps. Indeed, different methods have different objectives and possibilities, in line with the specific stochastic problem they set out to solve best. Each component separation method produces at least a CMB map, a confidence map (i.e., a mask), an effective beam, and a noise estimate map, together characterising that CMB map. Their (lack of) difference allows to probe which features are truly resilient. Figure 1 illustrates the results of one of them (here SMICA).

\subsection{CMB map isotropy and general non-gaussian statistics}

The previous CMB maps may be used to study the statistical isotropy and Gaussianity of the CMB. Here I survey null-hypothesis testing: a number of tests are performed, then p-values are calculated. A posteriori correction for "look-elsewhere effect" was adressed whenever possible. However, it is in the very nature of such a model-independent approach to leave the interpretation to further research.

All of the results we obtained are robust with respect to the choice of component separated CMB map. This is important since it demonstrates the high quality and equivalence for that purpose of the Planck component-separated data products rendered by different methodologies under varying assumptions.

It is found that the CMB is largely consistent with statistical isotropy, although there are a few indications of anomalies with respect to the expectations of $\Lambda \mathrm{CDM}$. Some of the tests performed in 2015 are the same as those in the 2013 release, in which case the results are consistent. Since many of these anomalies were also observed in the WMAP temperature data, the agreement between the two independent experiments effectively rules out the possibility that the origin of these features can be found in residual systematic artefacts present in either data set (either originating from the instruments or foregrounds).

Aspects of the statistics of the CMB fluctuations were assessed with tests of skewness, kurtosis, multinormality, N-point functions, and Minkowski functionals, and none yielded indications of significant departures from Gaussianity, while the variance of the CMB map was found to be low, in agreement with previous studies. First-order moments of filtered maps also exhibit the low variance anomaly, as well as a kurtosis excess on certain scales associated with the so called "Cold Spot". A study of peak statistics finds results consistent with the expectations for a Gaussian random field, although the Cold Spot is again detected.

The low variance anomaly appears to be associated with the known low- $\ell$ deficit in the angular power spectrum (see $\$ 3.3$ below). The lack of large-scale angular correlations, the relatively featureless northern ecliptic hemisphere 3- and 4-point functions, and indications of violations of point- and mirror-parity symmetry are also confirmed (although little attempt was made to correct these for a posteriori effects). Tight constraints on a quadrupolar power modulation were also obtained.

The now well-known large-scale dipolar power asymmetry (at 7\% level) was given particular attention. This asymmetry was detected via pixel-to-pixel variance, as well as by measuring power explicitly or indirectly via $\ell$ to $\ell \pm 1$ mode coupling. The latter approach lends itself to a posteriori correction, which reduces the significance of the asymmetry substantially when no model for the anomaly is assumed. In addition, two independent but related tests of directionality were conducted: one finds suggestions of anomalous clustering of directions out to relatively small 
scales while the other does not, evidently due to being optimized for slightly different forms of directionality.

Finally, stacking of temperature and polarization peaks was analysed. They are largely consistent with statistically isotropic simulations, both for oriented and unoriented stacking. The exception is a low unoriented temperature profile, which seems to be yet another reflection of the large-scale power deficit.

With the Planck 2015 release, one is probably near the limit of our ability to probe the CMB anomalies with temperature fluctuations alone. The use of large-angular-scale polarization, expected for the final Planck release in 2016, should enable independent tests of these peculiar features. Importantly, this will reduce or eliminate the subjectivity and ambiguity in interpreting their statistical significance. It is a tantalizing possibility that some of the anomalies described so far will take us beyond the standard model of cosmology. All of these results are detailed in [51].

\section{Power spectra from Planck}

The CMB angular power spectra contain all of the information available if the CMB is statistically isotropic and distributed as a multivariate Gaussian, which we now know is an excellent approximation. For realistic data, these empirical spectra must be augmented with models of instrumental noise, of various instrumental or processing systematic effects, and of contamination from astrophysical foregrounds. The CMB power spectra are in turn uniquely determined by the underlying cosmological model and its parameters. In temperature, the power spectrum has been measured over large fractions of the sky by COBE [64] and WMAP [3], and in smaller regions by a host of balloon- and ground-based telescopes $[e . g, 10 ; 11 ; 21-23 ; 26 ; 28 ; 39 ; 40 ; 58 ; 60 ; 62]$. The Planck 2013 power spectrum and likelihood were discussed in [44], and the 2015 analysis may be found in [53].

Over the last decade, CMB intensity (temperature) has been augmented by linear polarization data $[$ e.g $14 ; 31 ; 33 ; 55-57 ; 59]$. Because linear polarization is given by both an amplitude and direction, it can in turn be decomposed into two coordinate-independent quantities with different dependence on the cosmology $[e . g, 27 ; 65]$. One, the so-called $E$ mode which is the curl-free part, is determined by much the same physics as the intensity, and therefore allows an independent measurement of the background cosmology, as well as an improved determination of some parameters (e.g., the reionization optical depth). The other polarization observable, the $B$ mode, is only sourced at early times by tensor modes (gravitational radiation), as produced for example during an inflationary epoch. The $E$ and $B$ components are also conventionally taken to be isotropic Gaussian random fields, with only $E$ expected to be correlated with intensity. Thus we expect to be able to measure four independent power spectra, namely the three auto-spectra $C_{\ell}^{T T}, C_{\ell}^{E E}$, and $C_{\ell}^{B B}$, along with the cross-spectrum $C_{\ell}^{T E}$.

The distribution of temperature and polarization on the sky is further affected by gravitational lensing by the inhomogeneous mass distribution along the line of sight between the last scattering surface and the observer. This introduces correlations between large and small scales, which can be gauged by computing the expected contribution of lensing to the 4-point function (i.e., the trispectrum). This can in turn be used to determine the power spectrum of the lensing potential, as is done in [50] for the 2015 Planck release, and to further constrain the cosmological parameters 

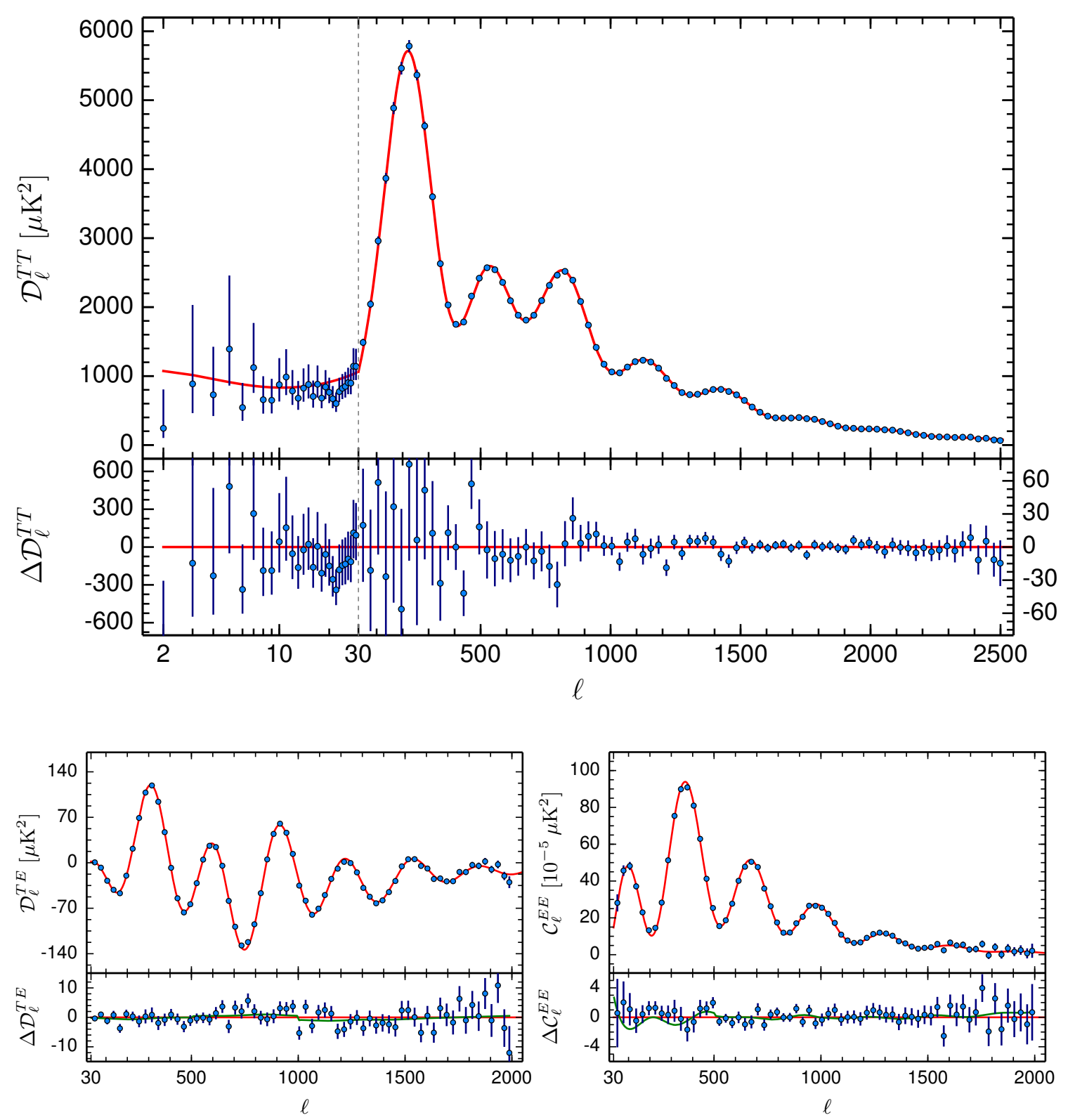

Figure 2: Planck 2015 CMB power spectra of TT (top), TE and EE (bottom), compared with the base LCDM fit (red line). The upper panels show the binned spectra and the lower panels the residuals of the fit. For all plots, the horizontal scale changes from logarithmic to linear at the hybridization scale, $\ell=29$. For the residuals, the vertical axis scale changes as well, shown by different left and right tick marks. Note that we show $\mathcal{D}_{\ell}=\ell(\ell+1) C_{\ell} /(2 \pi)$ for TT and TE but $C_{\ell}$ for EE, which also has different vertical scales at lowand high- $\ell$. 
via a separate likelihood function [48]. The following paragraphs provide an introduction to both (2-pt and lensing 4-pt). The lensing effect, in addition to modifying the $\mathrm{T}$ and $\mathrm{E}$ fields, generates $B$ modes (from the initial $E$ ones) which superimpose on any possible primordial ones.

\subsection{CMB power spectra from Planck}

The Planck paper [53] obtains the $C_{\ell}^{T T}, C_{\ell}^{E E}$, and $C_{\ell}^{T E}$ spectra (Fig. 2), likelihood functions, and basic cosmological parameters from the 2015 release. It shows that the contribution of high- $\ell$ systematic errors to the polarization spectra are at quite a low level (of the order of a few $(\mu \mathrm{K})^{2}$ ), therefore allowing an interesting comparison of the polarization-based cosmological results with those derived from $C_{\ell}^{T T}$ alone. Planck presented results for $C_{\ell}^{T E}$ and $C_{\ell}^{E E}$ at high multipoles. However, the technical difficulties involved with polarization measurements and subsequent data analysis, along with the inherently lower signal-to-noise ratio (especially for $B$ modes), thus require a careful understanding of the random noise and instrumental and astrophysical systematic effects which is less definitive than in temperature. For this reason, at large angular scales (i.e., low multipoles $\ell$ ) the 2015 baseline results use only a subset of Planck polarization data.

Because of these different sensitivities to systematic errors at different angular scales, as well as the increasingly Gaussian behaviour of the likelihood function at smaller angular scales, Planck adopted a hybrid approach to the likelihood calculation [15; 16], splitting between a direct calculation of the likelihood on large scales and the use of pseudo-spectral estimates at smaller scales.

At low multipoles, the current Planck release implements a standard joint pixel-based likelihood including both temperature and polarization for multipoles $\ell \leq 29$. For temperature, the formalism uses the cleaned Commander [18; 19] maps, while for polarization we used the $70 \mathrm{GHz}$ LFI maps ${ }^{3}$ and explicitly marginalize over the $30 \mathrm{GHz}$ and $353 \mathrm{GHz}$ maps taken as tracers of synchrotron and dust emission, respectively, accounting in both cases for the induced noise covariance in the likelihood.

At high multipoles $(\ell>29)$, as in [44], we use a likelihood function based on pseudo- $C_{\ell} \mathrm{s}$ calculated from Planck HFI data, as well as further parameters describing the contribution of foreground astrophysical emission and instrumental effects (e.g., calibration, beams). Aside from the processing improvements of the data themselves, the main advances over 2013 include the use of high $\ell$ polarization information along with more detailed models of foregrounds and instrumental effects. We constructed our likelihood approximation at high- $\ell$ by compressing all of the individual Planck detector data into mask-corrected (pseudo-) cross-spectra, and built a grand likelihood using these spectra and the corresponding analytical covariance matrix:

$$
-\ln \mathcal{L}(\hat{C} \mid C(\theta))=\frac{1}{2}[\hat{C}-C(\theta)]^{T} \mathbf{C}^{-1}[\hat{C}-C(\theta)]+\text { const },
$$

where $\hat{C}$ is the data vector, $C(\theta)$ is the model with parameters $\theta$, and $\mathbf{C}$ is the covariance matrix. Note that this formalism allows to separately marginalize over or condition upon different components of the model vector, separately treating cases such as individual frequency-dependent spectra, or temperature and polarization spectra. Obviously, Planck maps at different frequencies have different constraining powers on the underlying CMB, and we used this to impose and assess various

\footnotetext{
${ }^{3}$ The HFI data, having much less noise, therefore requires a much tighter control of any residual systematic effect, and we preferred to defer this more powerful but delicate analysis to a later release.
} 
cuts to keep only the most relevant data in the data vector. Indeed, we retained only the three best CMB Planck channels, i.e., $100 \mathrm{GHz}, 143 \mathrm{GHz}$, and $217 \mathrm{GHz}$, in the multipole range where they have significant $\mathrm{CMB}$ contributions and low enough foreground contamination after masking. Further, in order to achieve a significant reduction in the covariance matrix size (and computation time), we compressed the data vector (and accordingly the covariance matrix), both by co-adding the individual detectors for each frequency and by binning the combined power spectra.

The construction of a Gaussian approximation to the likelihood function requires building covariance matrices for the pseudo-power spectra. Mathematically exact expressions exist, but they are prohibitively expensive to calculate numerically at Planck resolution [63]; we thus made use of analytical approximations $[9 ; 15 ; 24 ; 25]$. For our baseline likelihood, we calculate covariance matrices for all 45 unique detector combinations that can be formed out of the six frequency-averaged half-mission maps at 100,143, and $217 \mathrm{GHz}$. To do so, we assume a fiducial power spectrum that includes the data variance induced by the CMB and all identified foreground components (which allow a good description of all relevant Planck data, including at frequencies not used in the likelihood); this variance is computed assuming these components are Gaussian-distributed. The effect of this approximation regarding Galactic foregrounds was shown negligible by means of simulations. The fiducial model is taken from the best-fit cosmological and foreground parameters; since they only become available after a full exploration of the likelihood, we iteratively refined our initial guess.

The resulting spectra are shown in Fig. 2, with the full statistical description being embodied in a likelihood code (applied to a set of empirical spectra from Planck) which numerically returns the likelihood of an input set of theoretical CMB spectra, and accounts for relevant uncertainties both instrumental and astrophysical in nature (as well as the correlations induced by the specific processing of the data). This code is publicly available. With this release, Planck now detects 36 extrema in total, consisting of 19 peaks and 17 troughs. The figure also shows the best-fit base$\Lambda \mathrm{CDM}$ model obtained from TT data alone (red lines), which is a sufficient description for all spectra.

Detailed checks of consistency and null tests lead us to conclude that there might still be lowlevel systematics residuals in the $E$ polarisation spectra which are not yet fully unaccounted for, at the $O\left(1 \mu \mathrm{K}^{2}\right)$, an example of which is shown by the green lines in the bottom panel. We therefore advised against using this polarisation information for testing non-minimal models differing from more standard cases by wiggles of that order of magnitude. Nevertheless, the high-multipole polarization spectra from Planck are already good enough to allow a separate high-accuracy determination of the parameters of the $\Lambda \mathrm{CDM}$ model, showing consistency with those established independently from temperature information alone. As a graphical example of this consistency, the bottom panel of Fig.2 shows in red, superimposed to the data, the predicted TE and EE spectra within the $\Lambda \mathrm{CDM}$ model with the best parameters obtained on TT alone (also shown in red in the top TT panel). The fit is indeed very good, which is confirmed by more detailed statistical analysis.

One way of assessing the constraining power contained in a particular measurement of CMB anisotropies is to determine the effective number of $a_{\ell m}$ modes that have been measured. This is equivalent to estimating 2 times the square of the total $\mathrm{S} / \mathrm{N}$ in the power spectra, a measure that contains all the available cosmological information if we assume that the anisotropies are purely Gaussian (and hence ignore all non-Gaussian information coming from lensing, the CIB, cross- 


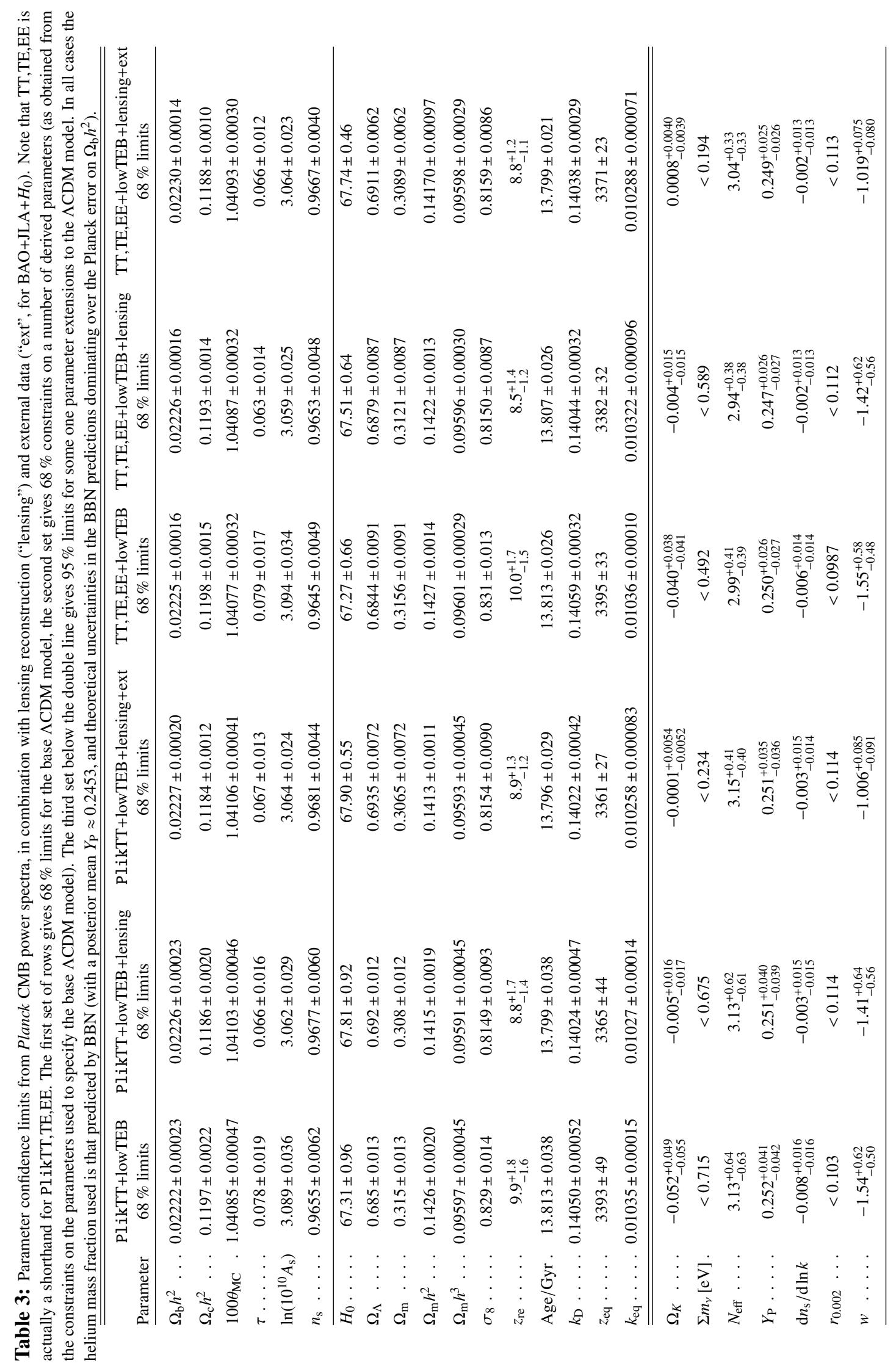


correlations with other probes, etc.). Carrying out this procedure for the Planck 2013 TT power spectrum data yields the number 826000 (which includes the effects of instrumental noise, cosmic variance and masking). The 2015 TT data have increased this value to 1114000 (in large part due to the increased fraction of the sky used), with $T E$ and $E E$ adding a further 60000 and 96000 modes, respectively. From this perspective the 2015 Planck data constrain approximately $55 \%$ more modes than in the 2013 release. Of course this is not the whole story, since some pieces of information are more valuable than others, and in fact Planck is able to place considerably tighter constraints on particular parameters (e.g., reionization optical depth or certain extensions to the base $\Lambda$ CDM model) by including new polarization data.

\section{2 $\Lambda$ CDM constraints from Planck CMB spectra alone}

The base (minimal) $\Lambda \mathrm{CDM}$ model with 6 parameters provides excellent fit to the data, including now in polarisation. The numerical values of the Planck 2015 cosmological parameters for $\Lambda \mathrm{CDM}$ are given in Table 3 (columns 2 and 5) in both cases. The tightening of the constraints when polarisation information is added to the temperature one is relatively modest for base $\Lambda \mathrm{CDM}$ (i.e., without considering any extension of the minimal 6 parameters model), due to the fact that they access mostly the same information for $\Lambda \mathrm{CDM}$, albeit through different physical mechanisms. Maybe more impressive is actually the fact that high- $\ell$ temperature-polarisation correlation in TE is already providing constraints which are of comparable precision for most $\Lambda \mathrm{CDM}$ parameters than those arising from the corresponding TT part. Not only does this offers a strong confirmation of the basic physics at play when not so long ago we had no single experiment constraining all the basic parameters, but it also constrains rather tightly potential extensions, like the existence of primordial isocurvature modes in addition to the dominating adiabatic ones.

We verified that degeneracies between foreground and calibration parameters generally do not affect the determination of the cosmological parameters. We further note that power spectra and parameters derived from CMB maps obtained by the component-separation methods described above and in [46] are generally consistent with those obtained here, at least when restricted to the $\ell<2000$ range in TT where they can be trusted for that purpose. We also checked that the derived foreground properties are consistent with current astrophysical knowledge. Of course, we also verified the consistency with the results from other CMB experiments.

\subsection{The low- $\ell$ "anomaly"}

In [44] we noted that the Planck 2013 low- $\ell$ temperature power spectrum exhibited a tension with the Planck best-fit model, which is mostly determined by high- $\ell$ information. In order to quantify such a tension, we performed a series of tests, concluding that the low- $\ell$ power anomaly was mainly driven by multipoles between $\ell=20$ and 30, which happen to be systematically low with respect to the base model. The statistical significance of this anomaly was found to be around $99 \%$, with slight variations depending on the Planck CMB solution or the estimator considered. This anomaly has drawn significant attention as a potential tracer of new physics (e.g., [13; 29; 30]; see also [12]), so we checked again its status in the 2015 data rendition.

Using a statistical measure (based on the Hausman test) of the relative bias between the observed spectrum at low- $\ell$ and a model, we found that the significance of that test for the Commander 
map has weakened from $0.7 \%$ in 2013 to $2.8 \%$ in 2015. This arises from small changes between the 2013 and 2015 Commander power spectra due to revised calibration and improved analysis on a larger portion of the sky. We also found with the same test that the new Planck low- $\ell$ polarization maps are anomalous only at the $7.7 \%$ level. The significance of this specific low- $\ell$ "anomaly" has therefore not been strengthened with the inclusion of more, better processed data, but it remains in the list of all the other, possibly related, large scale anomalies which we mentionned earlier.

\subsection{Planck Lensing power spectrum}

Lensing of the CMB photons by large scale structures on their path to the observer slightly distorts the image imprinted at the last scaterring surface. This has several effects. One is to slightly smooth the peak and trough structure of the CMB power spectra (which is fully accounted for by the numerical codes when deriving the parameter constraints on a model). Another one is to transform some of the polarisation E-modes into B-modes, adding to the potentially pre-existing Bmodes contribution from primordial tensor fluctuations. These distortions couple adjacent $\ell$ modes which would otherwise be uncorrelated if the initial fluctuations where statistically homogeneous (which is expressed by requiring translational invariance of the correlations). This can then be used to obtain an estimator of the lensing potential by cross-correlating CMB maps ( $T, E, B)$ smoothed at different scales.
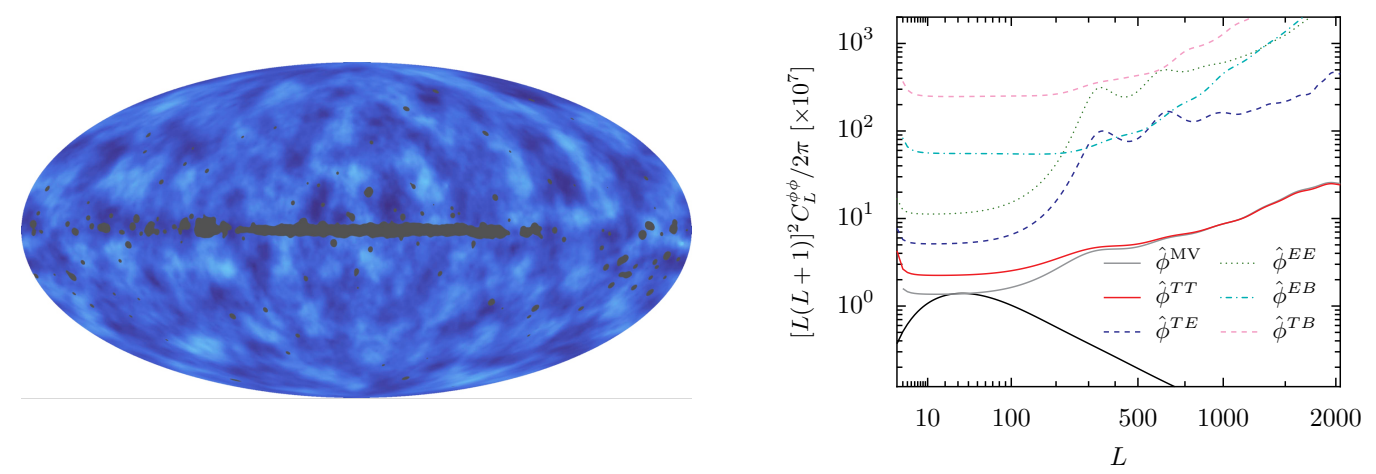

Figure 3: a) Wiener-filtered lensing potential estimate with minimal masking (using the NILC component separated map), in Galactic coordinates with a Mollweide projection [50]. The reconstruction has been bandlimited to $8 \leq L \leq 2048$ where $L$ is the multipole index in the lensing power spectrum). b) Lens reconstruction noise levels $N_{L}^{\Phi \Phi}$ (on top of the signal) for the TT, TE, EE, EB, and TB estimators applied to the SMICA full-mission CMB map. The noise level for their minimum-variance combination (MV) is also shown. The fiducial $\Lambda \mathrm{CDM}$ theory power spectrum $L$ used in our Monte Carlo simulations (to estimate biases) is plotted as the black solid line.

Figure 3 shows at left the resulting map of the estimated lensing potential, an integral along the line of sight of the gravitational potential weighted by a broad distance dependent kernel (which peaks at a redshift between 1.5 and two). This map is rather noisy. Indeed, Fig. 3 shows at right, in grey, the corresponding power spectrum (which is therefore a tri-spectrum that at a given multipole $L$ is obtained form the weighted product of four map harmonic coefficients $a_{\ell m}$ ). The black curve shows the predicted (noiseless) spectrum in the Planck base $\Lambda$ CDM model, the red curve shows the power spectrum obtained from a lensing map deriving from a temperature map, and the other curves the noisier reconstruction using polarisation data. Even for the minimum variance combination 
in grey, only a few large scale modes around $L \sim 20-50$ have a signal to noise comparable to one! Debiasing from the noise contribution therefore requires a quite accurate estimate of its value though detailed Monte-Carlo simulation.

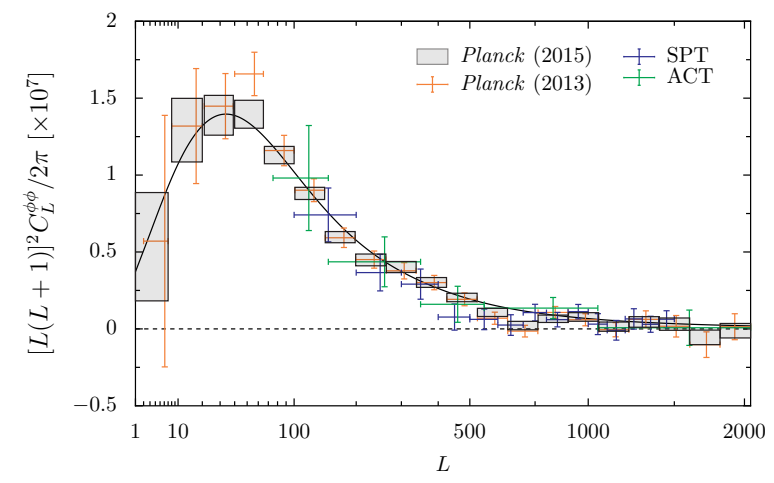

Figure 4: Lensing potential power spectrum estimate from the 2015 data release [50], based on the SMICA CMB map, as well as previous reconstructions from Planck as well as other experiments for comparison.

Figure 4 shows the final (debiased) result as greyed boxes, compared with all other determinations obtained so far. Planck for the first time measured the lensing power spectrum with higher accuracy than it is predicted by the base $\Lambda \mathrm{CDM}$ model that fits the temperature data (when the uncertainties of the fit are propagated forward). The amplitude is constained to about $2.5 \%$, a $40 \sigma$ detection for this lensing effect. The columns 3 and 6 of Table 3 show the improvements with respect to the already discussed columns 2 and 5 when Planck lensing is used in conjunction with Planck CMB power spectra in constraining the base $\Lambda$ CDM parameters. The improvement is modest for the base model where there is no spatial curvature and the dark energy equation of state is $w=-1$, two areas where the lower-z origin of lensing actually helps at lifting the degeneracies inherent to CMB only constraints (as can be seen in the bottom part of the table).

\section{5 $\Lambda$ CDM 1-parameter extensions and constraints from additional data}

The Planck paper dedicated to cosmological parameters [48] considered many other astrophysical sources of information, pertaining to Baryonic Acoustic Oscillations (BAO), Type-Ia supernovae (JLA, for Joint Light-curve Analysis), the current Hubble constant ${ }^{4}\left(H_{0}\right)$, as well as Planck cluster counts, redshift space distortions, and weak gravitational lensing. The first three, jointly refered to "ext", were found to be fully consistent with Planck CMB + lensing information within $\Lambda \mathrm{CDM}$, and the columns 4 and 7 of Table 3 gives the improvements they bring to the previous constraints. The other data sets, which generically constrain the amplitude of matter fluctuations at low z, exhibit some tension. These data are also notoriously difficult to analyse. New data sets should soon be available to suggest the most likely origin(s) of these tensions, systematic effects or physics beyond base $\Lambda$ CDM.

The base $\Lambda$ CDM model assumes an Friedman-Lemaître-Robertson-Walker metric with a flat spatial geometry. This is a very restrictive assumption which can now be tested empirically quite precisely. For $\Lambda \mathrm{CDM}$ models, the curvature parameter $\Omega_{K} \equiv 1-\Omega_{m}-\Omega_{\Lambda}$. A non zero detection

\footnotetext{
${ }^{4}$ The Planck "ext" analysis relies only on the $H_{0}$ prior derived in [17] by reanalysing Cepheid data using the revised geometric maser distance to NGC 4258 of Humphreys et al. (2013).
} 


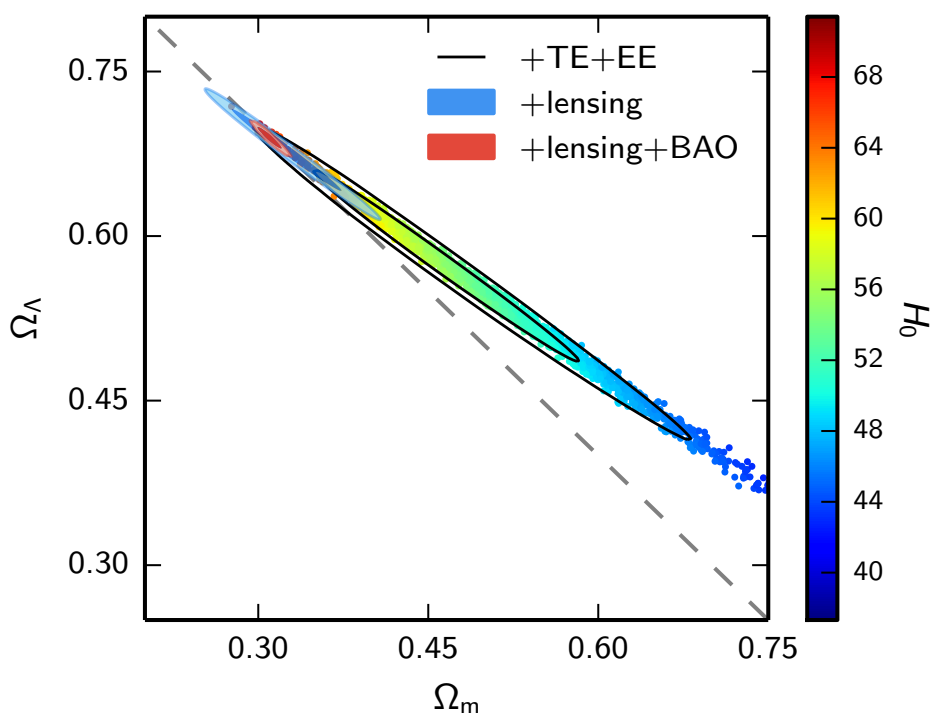

Figure 5: Constraints in the $\Omega_{m}-\Omega_{\Lambda}$ plane from the Planck TT+lowP data (samples; colour-coded by the value of $H_{0}$ ) and PlanckTT,TE,EE+lowP (solid contours). The geometric degeneracy between $\Omega_{\mathrm{m}}$ and $\Omega_{\Lambda}$ is partially broken because of the effect of lensing on the temperature and polarization power spectra. These limits are improved significantly by the inclusion of the Planck lensing reconstruction (blue contours) and BAO (solid red contours). The red contours tightly constrain the spatial geometry of our Universe to be nearly flat.

of $\Omega_{K}$ would surely have far-reaching implications for cosmology. Figure 5 illustrate graphically the tightening of the constraint with Planck CMB spectra, lensing, and external data sets in various combinations which are given numerically in Table 3. While the Planck CMB spectra remain affected by the "geometric degeneracy" 5 " $7 ; 66]$, the addition of Planck lensing leads to order of magnitude improvement on possible deviation of $\Omega_{K}$ from zero, which is further improved by including BAO data, yielding

$$
\begin{aligned}
& \Omega_{K}=-0.052_{-0.055}^{+0.049}(95 \%, \text { Planck TT+lowP }) \\
& \Omega_{K}=-0.005_{-0.017}^{+0.016}(95 \%, \text { Planck TT+lowP+lensing }) \\
& \Omega_{K}=0.000 \pm 0.005(95 \%, \text { Planck TT+lowP+lensing+BAO }) .
\end{aligned}
$$

The bottom part of Table 3 summarizes further constraints on single parameter extensions to the base $\Lambda \mathrm{CDM}$ model on, e.g., neutrinos properties $\Sigma m_{v}$ and $N_{\mathrm{eff}}$, the primordial Helium fraction, $Y_{\mathrm{P}}$ (when the standard BBN value is not assumed to hold), or the dark energy equation of state, $w=p / \rho$ (the cosmological constant corresponding to the case $w=-1$ ).

Regarding the constraints on initial conditions, it is convenient to expand the power spectra of primordial curvature and tensor perturbations on super-Hubble scales as

$$
\mathcal{P}_{\mathcal{R}}(k)=A_{\mathrm{s}}\left(\frac{k}{k_{*}}\right)^{n_{\mathrm{s}}-1+\frac{1}{2} \mathrm{~d} n_{\mathrm{s}} / \mathrm{d} \ln k \ln \left(k / k_{*}\right)+\ldots}, \quad \mathcal{P}_{\mathrm{t}}(k)=A_{\mathrm{t}}\left(\frac{k}{k_{*}}\right)^{n_{\mathrm{t}}+\frac{1}{2} \mathrm{~d} n_{\mathrm{t}} / \mathrm{d} \ln k \ln \left(k / k_{*}\right)+\ldots},
$$

\footnotetext{
${ }^{5}$ This degenary allows for the small-scale linear CMB spectrum to remain almost unchanged if changes in $\Omega_{K}$ are compensated by changes in $H_{0}$ to obtain the same angular diameter distance to last scattering.
} 


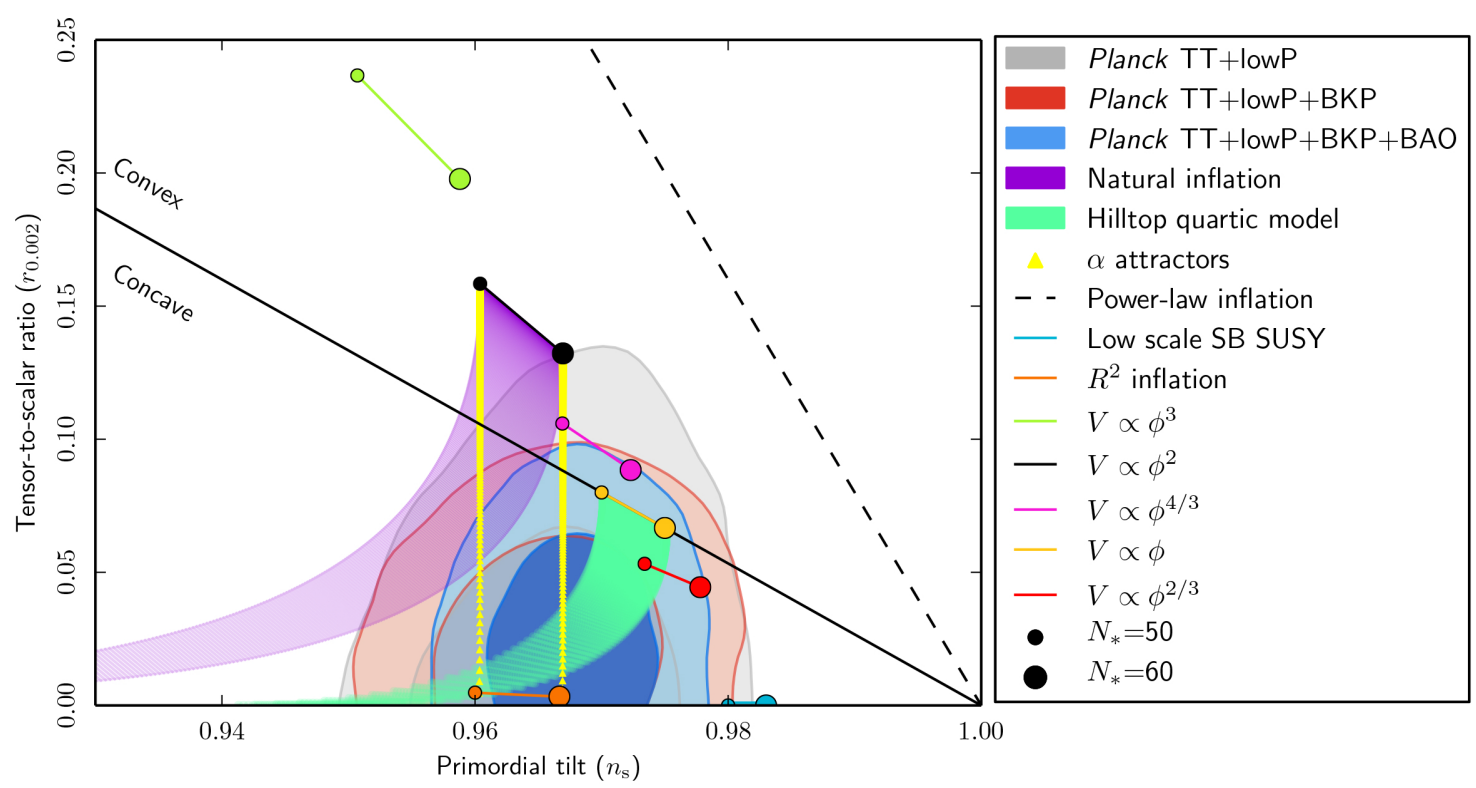

Figure 6: Marginalized joint $68 \%$ and $95 \% \mathrm{CL}$ regions for $n_{\mathrm{s}}$ and $r_{0.002}$ from Planck alone and in combination with other data sets (Bicep2+Keck cross Planck, aka, BKP, and BAO, bottom), compared to the theoretical predictions of selected inflationary models.

where $A_{\mathrm{s}}\left(A_{\mathrm{t}}\right)$ is the scalar (tensor) amplitude and $n_{\mathrm{S}}\left(n_{\mathrm{t}}\right)$, and $\mathrm{d} n_{\mathrm{s}} / \mathrm{d} \ln k\left(\mathrm{~d} n_{\mathrm{t}} / \mathrm{d} \ln k\right)$ are the scalar (tensor) spectral index, and the running of the scalar (tensor) spectral index, respectively. Fig. 6 shows the Planck joint constraints on $\left(n_{\mathrm{s}}, r_{0.002}\right)$, where $r_{0.002}$ stands for the tensor-to-scalar ratio at the pivot scale $k=0.002 \mathrm{Mpc}^{-1}$ from Planckalone and with other datasets, including the co-analysis with Bicep/Keck (see below). The plot also shows the expected parameter range for selected inflation models, when the number of e-folds to the end of inflation after the current Hubble scale froze out, $N_{\star}$, is in the standard range 50 to 60 . This plot shows that the canonical polynomial potential $V \propto \varphi^{2}$ is increasingly disfavoured and, more generically, all convex potentials as well (therefore favouring slow-roll models for which both $\dot{\varphi}$ and $\dot{\varphi} / \rho$ increase with time).

The numerical constraints on the tensor-to-scalar ratio $r_{0.002}$ (and on possible curvature of the scalar spectrum, $\mathrm{d} n_{\mathrm{s}} / \mathrm{d} \ln k$ ) using different data combinations are given in Table 3. Let us note that the constraint $r_{0.002}<0.11$ at $95 \% \mathrm{CL}$ which arises from Planck $\mathrm{TT}+$ lowP+lensing (and which is not really improved when adding external data, or high- $\ell$ polarisation) can hardly get tighter by using only $\mathrm{T} \& \mathrm{E}$ data. Indeed, $\mathrm{T} \& \mathrm{E}$ fluctuations are both sourced by scalar and tensor fluctuations, and residual degeneraries cannot be lifted without further information. Let us further note that the 2013 Planck constraint on $r$ was already quite similar.

\subsection{Co-analysis of Bicep2 and Planck data}

This is in this context that the Bicep2 collaboration announced worlwide, on the $17^{\text {th }}$ of March 2014, that it had detected the long-sought after microwave B-modes, an extraordinary result from a beautiful, extremely sensitive, experiment. This thus ended a long series of ever tighter upper limits on the B-modes power spectrum. They further claimed that the search for primordial gravitationnal 
waves was over, that $r=0.2$, and that it was a $5 \sigma$ detection!
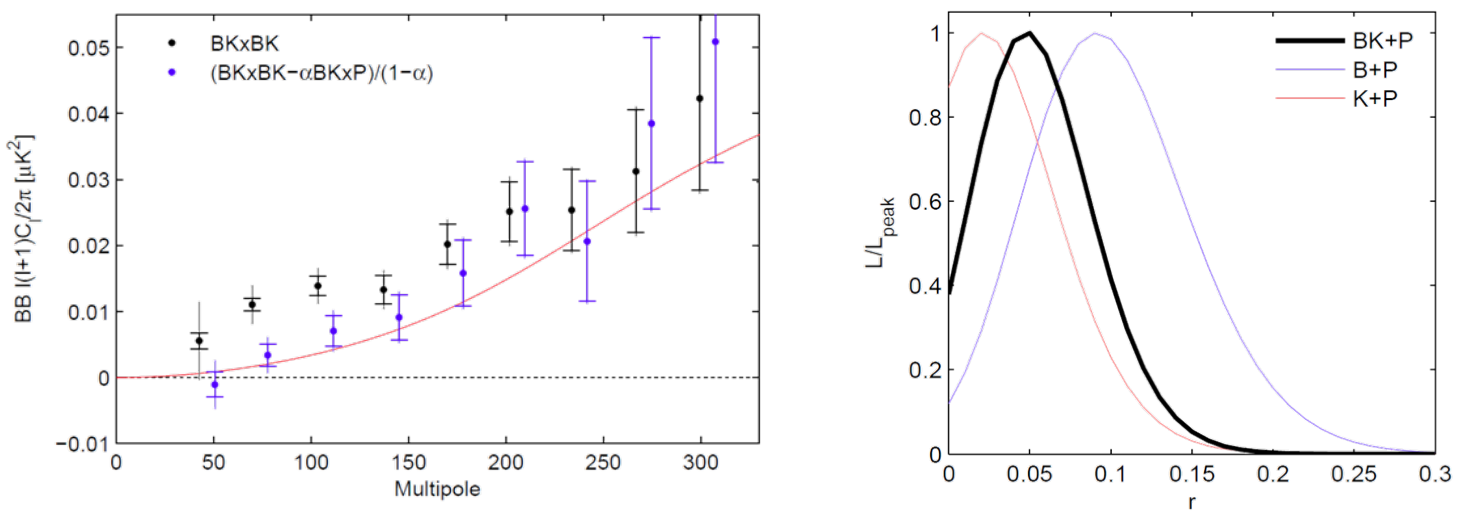

Figure 7: Results of the co-analysis of Planck and Bicep/Keck (BK) data. The left panel shows B-modes power spectra, both dust-correct (blue points) and uncorrected (black points), as well as the expected Bmodes from lensing of the E-modes (given in red). The right panel shows the likelihood of $r$ values, leading to the quoted $95 \%$ CL upper limit of $r_{0.05}<0.11$.

It was nevertheless pointed out extremely rapidly that a more mundane interpretation was possible [20;37; 41]. Indeed, the $5 \sigma$ detection of $r$ relied on the assumption that the polarised emission from Galactic dust was not contributing significantly at that frequency in that field, while the analysis presented could not exclude that it was actually all coming from such dust emission, even at the $3 \sigma$ level (for lack of sufficient spectral information, [2]). And indeed we published shortly after a paper showing, on the basis of the all-sky $353 \mathrm{GHz}$ Planck data (used as a tracer for polarised dust emission) that about half of the detected B-modes in that field was expected to be due to dust emission, albeit with sizeable uncertainties. It was indeed not possible to be very precise without having access to detailed non-public information like the weights of the BICEP2 pixels in the final results. The two collaborations therefore performed a joint analysis [6], in particular by directly cross-correlating the Planck $353 \mathrm{GHz}$ data and the $145 \mathrm{GHz}$ data from Bicep (and the additional Keck data from the same collaboration, noted below as BK) to assess the dust contamination level and shape.

The main results of the joint analysis are shown in Fig. 7. The left panel displays B-modes power spectra. The black point correspond to the uncorrected spectrum determination $(\mathrm{BK} \times \mathrm{BK})$ which shows the basis of the claimed detection as a clear excess with respect to the expected Bmodes from lensing given by the red curve. The blue points shows the dust-corrected result with the help of Planck data, which is consistent with the predicted level from the lensing of E-modes and actually offers a beautifully precise determination of the later (at the $0.1 \mu \mathrm{K}$ level!). The right panel displays the likelihood of $r$ when either Bicep2 (B), or Keck (K), or both datasets (BK) are cleaned with Planck data $(\mathrm{P})$. There is no clear evidence left for primordial B-modes, and we quoted the upper limit $r_{0.05}<0.11$ at $95 \% \mathrm{CL}$, or equivalently $r_{0.005}<0.12$. This direct upper limit on $r$ is now completely compatible with the indirect limit set previously by Planck in 2013, removing the need for less minimal model than base $\Lambda$ CDM. Fig. 6 shows the further tightening obtained when Planck and BKP $(\mathrm{BK} \times$ Planck) constraints are combined (The combined constraint Planck $\mathrm{TT}+$ lowP+BKP yields $r_{0.002}<0.08$ at $95 \% \mathrm{CL}$ ). This is the state of the art. 


\subsection{Exploring further degrees of freedom}

Table 3 already gave constraints on possible curvature of the scalar spectrum, $\mathrm{d} n_{\mathrm{s}} / \mathrm{d} \ln k$, but that single extra parameter would not allow capturing well, or at all, many possible features of the potential or or the primordial power spectrum, $\mathcal{P}_{\mathcal{R}}$ (as for example a step in the inflaton potential or oscillations). The Planck paper [53] provides Bayesian evidences for a number of theoretically motivated parameterizations of the spectrum, but none of these features models are preferred over a power-law spectrum, for the choice of priors considered (and the constraints on the remaining cosmological parameters are not significantly affected when allowing for the presence of these features).
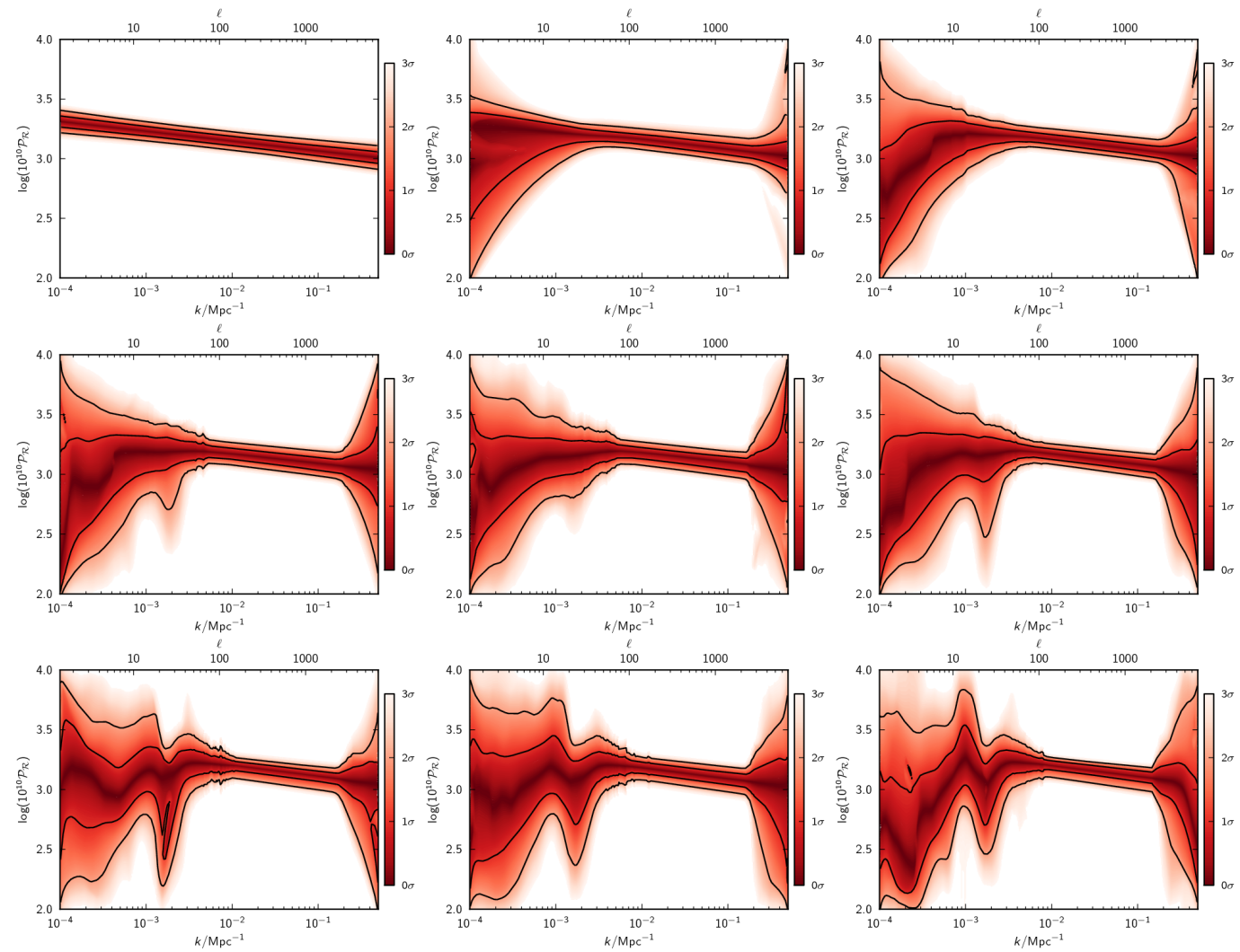

Figure 8: Bayesian movable knot reconstructions of the primordial power spectrum $\mathcal{P}_{\mathcal{R}}(k)$ using Planck TT+lowP data. The plots indicate our knowledge of the $\mathrm{P}\left(\mathcal{P}_{\mathcal{R}}(k) \mid k, N_{\mathrm{knot}}\right)$ for a given number of reconstruction knots, $N_{\text {knot }}$. The number of these knots $N_{\text {knot }}$ increases (left to right and top to bottom) from 2 to 10 . $1 \sigma$ and $2 \sigma$ confidence intervals are outlined (black curves). The upper horizontal axes give the approximate corresponding multipoles via $\ell \approx k / D_{\text {rec }}$, where $D_{\text {rec }}$ is the comoving distance to recombination.

Since the mapping from the primordial power spectrum to the angular power spectra is a linear transformation, one can unambiguously reconstruct some part of it. The Planck paper devoted to inflation [53] presents three such reconstruction. Figure 8 shows one of them, for an increasingly number of reconstruction knots assumed, from 2 knots (i.e., only a single power law is allowed, as in the standard analysis), to 10. These knots are freely movable. Equal colours have equal probabilities For each reconstruction and the colour scale is chosen so that darker regions corre- 
spond to lower- $\sigma$ confidence intervals. $1 \sigma$ and $2 \sigma$ confidence intervals are also sketched (black curves). The upper horizontal axes give the approximate corresponding multipoles via $\ell \approx k / D_{\text {rec }}$, where $D_{\text {rec }}$ is the comoving distance to recombination. Apart from a low $k$ deficit driven by the low quadrupole (for $N_{\mathrm{knot}}>2$ ), the main feature which is clearly visible with $\gtrsim 8$ knots is a power deficit corresponing to the anomaly of the TT spectrum at $\ell \sim 30$. But the rest is clearly scale-free, with $n_{\mathrm{s}} \approx 0.96$.

These reconstructions can also be averaged over different values of $N_{\text {knot }}$ weighted according to their respective Bayesian evidence. The region $30<\ell<2300$ is highly constrained, but the resolution is lacking to say anything precise about higher $\ell$. At lower $\ell$, cosmic variance reduces our knowledge of $\mathcal{P}_{\mathcal{R}}(k)$. The weights assigned to the lower $N_{\text {knot }}$ models outweigh those of the higher models, so no oscillatory features are significant there.

All three methods yield broadly consistent reconstructions and lead to conclude that there is no statistically significant evidence for any features departing from a simple power law (i.e., $\mathcal{P}_{\mathcal{R}}(k) \propto k^{n_{\mathrm{s}}-1}$ ) primordial spectrum. But at low $k \approx 1.5-2.0 \times 10^{-3} \mathrm{Mpc}^{-1}$, all three methods reconstruct a power deficit linked to the dip in the TT angular power spectrum at $\ell \sim 20-30$. This agreement suggests that the reconstruction of this "anomaly" is not an artefact of any of the methods, but rather inherent in the CMB data itself. However, the evidence for this feature is marginal since it is in a region of the spectrum where the fluctuations from cosmic variance are large.

\section{Primordial Non-Gaussianity}

Primordial non-Gaussianity (NG) is one of the most powerful tests of inflation, and more generally of high-energy early universe physics, in a way that is strongly complementary to the power-spectrum constraints already discussed. The simplest models of inflation, characterized by a single scalar field slowly rolling along a smooth potential, predict the generation of primordial fluctuations which are almost Gaussian distributed, with a tiny deviation from Gaussianity of the order of the slow-roll parameters $[1 ; 35]$. On the other hand any departure from these hypothese has the potential to produce distinctive NG signatures at a detectable level in the CMB anisotropies.

We used several analysis methods (e.g., the modal approach, as well as other estimators, (the optimal "KSW" with its skew-Cl extension [32; 38], wavelets, Minkowski functionals...), since these different weighting of the triple-product of modes would be affected differently by residual systematic effects. We have extensively tested for the latter using simulations and the 4 component separation methods of Planck, testing for the effect of masks and $\ell_{\max }$ of the analysis, and debiased them when needed, in particular to account for the ISW-lensing part or residual compact sources.

All approaches lead to the same conclusion, i.e., the Planck results [52] on primordial NG are consistent with Gaussian primordial fluctuations. In particular, using temperature only data,

$$
\begin{aligned}
& f_{N L}-\text { local }=+1.8 \pm 5.6 \\
& f_{N L}-\text { equil }=-9.2 \pm 69 \\
& f_{N L}-\text { ortho }=-20 \pm 33
\end{aligned}
$$


for the amplitudes of three of the most-studied shapes of primordial NG. These are by far the tighest constraints available on extensions to the simplest models of inflation: the standard scenario of single-field slow-roll inflation has survived its most stringent test to-date.

\section{Conclusions}

This contribution gave a short overview of the latest Planck data and findings of most interest for inflation, which are commented more in depth in [8]. A complete analysis in the context of an extended $\Lambda \mathrm{CDM}$ cosmology of these and other results from Planck regarding the lensing power spectrum results, as well as constraints from other observations, is given in [47]. Wider extensions to the set of models are discussed in other Planck 2015 papers; for example, [49] examines specific models for the dark energy component and [53] discusses inflationary models. The Planck final "legacy" data and analyses are being prepared for release in 2016, where the improvements will mostly concern the CMB polarisation.

\section{Acknowledgments}

All results presented are those of the Planck collaboration, as published or submitted. All mistakes on top are mine :-).

The development of Planck has been supported by: ESA; CNES and CNRS/INSU-IN2P3-INP (France); ASI, CNR, and INAF (Italy); NASA and DoE (USA); STFC and UKSA (UK); CSIC, MICINN, JA and RES (Spain); Tekes, AoF and CSC (Finland); DLR and MPG (Germany); CSA (Canada); DTU Space (Denmark); SER/SSO (Switzerland); RCN (Norway); SFI (Ireland); FCT/MCTES (Portugal); and PRACE (EU). A description of the Planck Collaboration and a list of its members, including the technical or scientific activities in which they have been involved, can be found at http://www . sciops . esa . int/index . php?project=planck\&page=Planck_ Collaboration.

\section{References}

[1] Acquaviva, V., N. Bartolo, S. Matarrese, and A. Riotto, Gauge-invariant second-order perturbations and non-Gaussianity from inflation, Nuclear Physics B, 667, 119-148, 2003.

[2] Ade, P. A. R., et al., Detection of B-Mode Polarization at Degree Angular Scales by BICEP2, Physical Review Letters, 112, 241,101, 2014.

[3] Bennett, C. L., et al., Nine-year Wilkinson Microwave Anisotropy Probe (WMAP) Observations: Final Maps and Results, ApJS, 208, 20, 2013.

[4] Bersanelli, M., et al., Planck pre-launch status: Design and description of the Low Frequency Instrument, $A \mathcal{E} A$, 520, A4, 2010.

[5] Béthermin, M., et al., A Unified Empirical Model for Infrared Galaxy Counts Based on the Observed Physical Evolution of Distant Galaxies, ApJ, 757, L23, 2012.

[6] BICEP2/Keck Array and Planck Collaborations, Joint Analysis of BICEP2/Keck Array and Planck Data, Phys. Rev. Lett., 114, 101,301, 2015.

[7] Bond, J., G. Efstathiou, and M. Tegmark, Forecasting cosmic parameter errors from microwave background anisotropy experiments, Mon.Not.Roy.Astron.Soc., 291, L33-L41, 1997. 
[8] Bouchet, F. R., Planck 2015 results and inflation, Comptes Rendus Physique, 16, 891 - 913, 2015, cosmic inflation / Inflation cosmique.

[9] Challinor, A., and G. Chon, Error analysis of quadratic power spectrum estimates for cosmic microwave background polarization: sampling covariance, MNRAS, 360, 509-532, 2005.

[10] Das, S., et al., The Atacama Cosmology Telescope: A Measurement of the Cosmic Microwave Background Power Spectrum at 148 and $218 \mathrm{GHz}$ from the 2008 Southern Survey, ApJ, 729, 62, 2011.

[11] Das, S., et al., The Atacama Cosmology Telescope: Temperature and Gravitational Lensing Power Spectrum Measurements from Three Seasons of Data, ArXiv e-prints, 2013.

[12] Destri, C., H. J. de Vega, and N. G. Sanchez, CMB quadrupole depression produced by early fast-roll inflation: Monte Carlo Markov chains analysis of WMAP and SDSS data, Phys. Rev. D, 78, 023,013, 2008.

[13] Dudas, E., N. Kitazawa, S. P. Patil, and A. Sagnotti, CMB imprints of a pre-inflationary climbing phase, J. Cosmology Astropart. Phys., 5, 12, 2012.

[14] Dunkley, J., et al., Five-Year Wilkinson Microwave Anisotropy Probe Observations: Likelihoods and Parameters from the WMAP Data, ApJS, 180, 306-329, 2009.

[15] Efstathiou, G., Myths and truths concerning estimation of power spectra: the case for a hybrid estimator, MNRAS, 349, 603-626, 2004.

[16] Efstathiou, G., Hybrid estimation of cosmic microwave background polarization power spectra, $M N$ RAS, 370, 343-362, 2006.

[17] Efstathiou, G., $\mathrm{H}_{0}$ revisited, MNRAS, 440, 1138-1152, 2014.

[18] Eriksen, H. K., I. J. O’Dwyer, J. B. Jewell, B. D. Wandelt, D. L. Larson, K. M. Górski, S. Levin, A. J. Banday, and P. B. Lilje, Power Spectrum Estimation from High-Resolution Maps by Gibbs Sampling, ApJS, 155, 227-241, 2004.

[19] Eriksen, H. K., J. B. Jewell, C. Dickinson, A. J. Banday, K. M. Górski, and C. R. Lawrence, Joint Bayesian Component Separation and CMB Power Spectrum Estimation, ApJ, 676, 10-32, 2008.

[20] Flauger, R., J. C. Hill, and D. N. Spergel, Toward an understanding of foreground emission in the BICEP2 region, J. Cosmology Astropart. Phys., 8, 39, 2014.

[21] Fowler, J. W., et al., The Atacama Cosmology Telescope: A Measurement of the $600<\ell<8000$ Cosmic Microwave Background Power Spectrum at 148 GHz, ApJ, 722, 1148-1161, 2010.

[22] Grainge, K., et al., The cosmic microwave background power spectrum out to $1=1400$ measured by the Very Small Array, MNRAS, 341, L23-L28, 2003.

[23] Hanany, S., et al., MAXIMA-1: A Measurement of the Cosmic Microwave Background Anisotropy on Angular Scales of 10 arcmin-5 degree, ApJ, 545, L5-L9, 2000.

[24] Hansen, F. K., K. M. Górski, and E. Hivon, Gabor transforms on the sphere with applications to CMB power spectrum estimation, MNRAS, 336, 1304-1328, 2002.

[25] Hinshaw, G., et al., First-Year Wilkinson Microwave Anisotropy Probe (WMAP) Observations: The Angular Power Spectrum, ApJS, 148, 135-159, 2003.

[26] Jones, W. C., et al., A Measurement of the Angular Power Spectrum of the CMB Temperature Anisotropy from the 2003 Flight of BOOMERANG, ApJ, 647, 823-832, 2006.

[27] Kamionkowski, M., A. Kosowsky, and A. Stebbins, Statistics of cosmic microwave background polarization, Phys. Rev. D, 55, 7368-7388, 1997.

[28] Keisler, R., et al., A Measurement of the Damping Tail of the Cosmic Microwave Background Power Spectrum with the South Pole Telescope, ApJ, 743, 28, 2011.

[29] Kitazawa, N., and A. Sagnotti, Pre-inflationary clues from String Theory?, J. Cosmology Astropart. Phys., 4, 17, 2014.

[30] Kitazawa, N., and A. Sagnotti, A String-Inspired Model for the Low-\$ $\backslash$ ell\$ CMB, ArXiv e-prints, 2015.

[31] Kogut, A., et al., First-Year Wilkinson Microwave Anisotropy Probe (WMAP) Observations: Temperature-Polarization Correlation, ApJS, 148, 161-173, 2003. 
[32] Komatsu, E., D. N. Spergel, and B. D. Wandelt, Measuring Primordial Non-Gaussianity in the Cosmic Microwave Background, ApJ, 634, 14-19, 2005.

[33] Kovac, J. M., E. M. Leitch, C. Pryke, J. E. Carlstrom, N. W. Halverson, and W. L. Holzapfel, Detection of polarization in the cosmic microwave background using DASI, Nature, 420, 772-787, 2002.

[34] Lamarre, J., et al., Planck pre-launch status: The HFI instrument, from specification to actual performance, $A \mathcal{E} A$, 520, A9, 2010.

[35] Maldacena, J., Non-gaussian features of primordial fluctuations in single field inflationary models, Journal of High Energy Physics, 5, 13, 2003.

[36] Mennella, A., et al., Planck early results. III. First assessment of the Low Frequency Instrument inflight performance, $A \mathcal{E} A, 536, \mathrm{~A} 3,2011$.

[37] Mortonson, M. J., and U. Seljak, A joint analysis of Planck and BICEP2 B modes including dust polarization uncertainty, J. Cosmology Astropart. Phys., 10, 35, 2014.

[38] Munshi, D., and A. Heavens, A new approach to probing primordial non-Gaussianity, MNRAS, 401, 2406-2418, 2010.

[39] Netterfield, C. B., M. J. Devlin, N. Jarosik, L. Page, and E. J. Wollack, A Measurement of the Angular Power Spectrum of the Anisotropy in the Cosmic Microwave Background, ApJ, 474, 47-66, 1997.

[40] Pearson, T. J., et al., The Anisotropy of the Microwave Background to $1=3500$ : Mosaic Observations with the Cosmic Background Imager, ApJ, 591, 556-574, 2003.

[41] Planck Collaboration, et al., Planck intermediate results. XXX. The angular power spectrum of polarized dust emission at intermediate and high Galactic latitudes, ArXiv e-prints, 2014.

[42] Planck HFI Core Team, Planck early results, IV. First assessment of the High Frequency Instrument in-flight performance, $A \mathcal{E} A, 536, \mathrm{~A} 4,2011$.

[43] Planck Collaboration I, Planck early results. I. The Planck mission, AEFA, 536, A1, 2011.

[44] Planck Collaboration XV, Planck 2013 results. XV. CMB power spectra and likelihood, AEA, 571 , A15, 2014.

[45] Planck Collaboration IV, Planck 2015 results. IV. LFI beams and window functions, AEA, in press, 2015.

[46] Planck Collaboration IX, Planck 2015 results. IX. Diffuse component separation: CMB maps, $A \mathcal{E} A$, submitted, 2015.

[47] Planck Collaboration XI, Planck 2015 results. XI. CMB power spectra, likelihoods, and robustness of parameters, AEFA, submitted, 2015.

[48] Planck Collaboration XIII, Planck 2015 results. XIII. Cosmological parameters, AEAA, submitted, 2015.

[49] Planck Collaboration XIV, Planck 2015 results. XIV. Dark energy and modified gravity, AEFA, submitted, 2015.

[50] Planck Collaboration XV, Planck 2015 results. XV. Gravitational lensing, AEA, submitted, 2015.

[51] Planck Collaboration XVI, Planck 2015 results. XVI. Isotropy and statistics of the CMB, $A \mathcal{E} A$, in press, 2015.

[52] Planck Collaboration XVII, Planck 2015 results. XVII. Constraints on primordial non-Gaussianity, AEFA, submitted, 2015.

[53] Planck Collaboration XX, Planck 2015 results. XX. Constraints on inflation, AEFA, submitted, 2015.

[54] Planck Collaboration XXVI, Planck 2015 results. XXVI. The Second Planck Catalogue of Compact

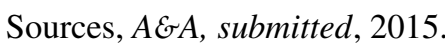

[55] Polarbear Collaboration, et al., A Measurement of the Cosmic Microwave Background B-mode Polarization Power Spectrum at Sub-degree Scales with POLARBEAR, ApJ, 794, 171, 2014.

[56] Pryke, C., et al., Second and Third Season QUaD Cosmic Microwave Background Temperature and Polarization Power Spectra, ApJ, 692, 1247-1270, 2009.

[57] QUIET Collaboration, et al., Second season quiet observations: Measurements of the cosmic microwave background polarization power spectrum at $95 \mathrm{ghz}, \mathrm{ApJ}, 760,145,2012$. 
[58] Reichardt, C. L., et al., High-Resolution CMB Power Spectrum from the Complete ACBAR Data Set, ApJ, 694, 1200-1219, 2009.

[59] Sievers, J. L., et al., Implications of the Cosmic Background Imager Polarization Data, ApJ, 660, 976-987, 2007.

[60] Story, K. T., et al., A Measurement of the Cosmic Microwave Background Damping Tail from the 2500-square-degree SPT-SZ survey, ArXiv e-prints, 2012.

[61] Tauber, J. A., et al., Planck pre-launch status: The Planck mission, AEAA, 520, A1, 2010.

[62] Tristram, M., et al., The CMB temperature power spectrum from an improved analysis of the Archeops data, $A \mathcal{E} A, 436,785-797,2005$.

[63] Wandelt, B. D., E. Hivon, and K. M. Górski, Cosmic microwave background anisotropy power spectrum statistics for high precision cosmology, Phys. Rev. D, 64, 083,003, 2001.

[64] Wright, E. L., C. L. Bennett, K. Górski, G. Hinshaw, and G. F. Smoot, Angular power spectrum of the cosmic microwave background anisotropy seen by the cobe dmr, The Astrophysical Journal Letters, 464, L21, 1996.

[65] Zaldarriaga, M., and U. Seljak, All-sky analysis of polarization in the microwave background, Phys. Rev. D, 55, 1830-1840, 1997.

[66] Zaldarriaga, M., D. N. Spergel, and U. Seljak, Microwave background constraints on cosmological parameters, Astrophys.J., 488, 1-13, 1997. 